\title{
Abordagem diagnóstica da síndrome braquicefálica em cães: revisão de literatura
}

\author{
Diagnostic approach to brachycephalic syndrome in dogs: literature review \\ Enfoque diagnóstico del síndrome braquicéfalo en perros: revisión de la literatura
}

Recebido: 02/11/2021 | Revisado: 10/11/2021 | Aceito: 15/11/2021 | Publicado: 24/11/2021

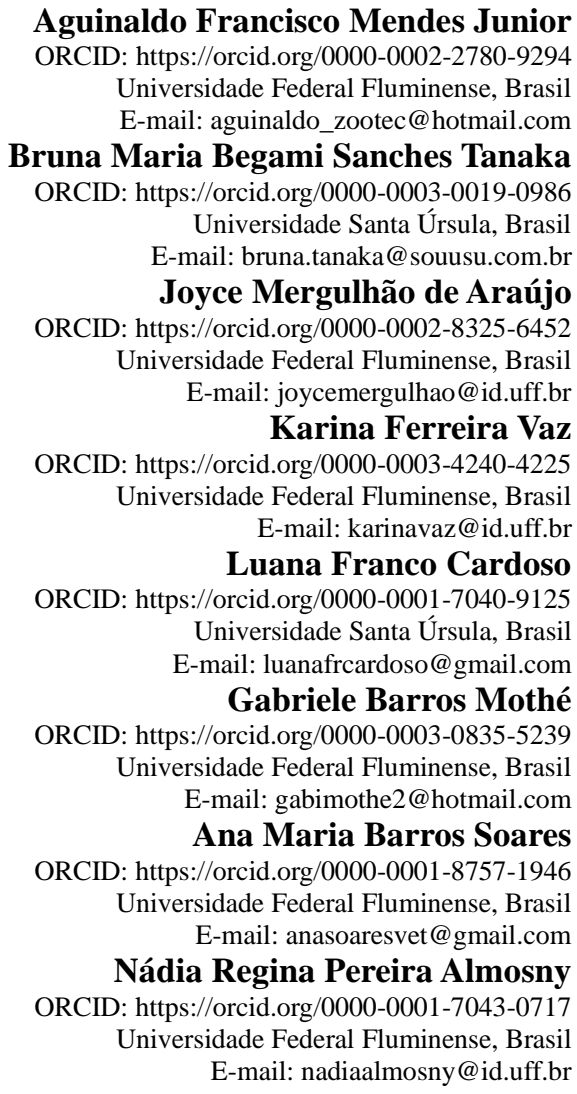

\begin{abstract}
Resumo
O reconhecimento precoce da síndrome braquicefálica é de extrema importância para um melhor prognóstico póscirúrgico, sendo primordial a associação de variados métodos de pesquisa para sua determinação. O diagnóstico da síndrome baseia-se em uma anamnese apurada juntamente com a observação dos sinais clínicos característicos e exames complementares, tais quais hematológicos e exames de imagem. Dentre os exames de imagem, são comumente utilizadas a radiografia, rinoscopia e tomografia computadorizada, que auxiliam tanto no diagnóstico da síndrome quanto no planejamento cirúrgico, quando indicado. Visto a importância do diagnóstico preciso desta enfermidade nos cães acometidos, o atual estudo objetivou realizar uma revisão bibliográfica atualizada abordando os diferentes métodos diagnósticos para a SB. Pode-se então concluir que a correta identificação da síndrome braquicefálica pelos médicos veterinários através das diferentes ferramentas diagnósticas é de extrema importância para estabelecer um tratamento precoce, minimizando o risco agudo de óbito e aumentando a qualidade de vida e bem estar dos animais acometidos.
\end{abstract}

Palavras-chave: Estenose de narinas; Prolongamento de palato mole; Laringoscopia; Rinoscopia.

\begin{abstract}
The early recognition of brachycephalic syndrome is extremely important for a better post-surgical prognosis, and the association of various research methods is essential for its determination. The diagnosis of the syndrome is based on an accurate anamnesis together with the observation of characteristic clinical signs and complementary exams, such as hematological exams and imaging exams. Among the imaging tests, radiography, rhinoscopy and computed tomography are commonly used, which help both in the diagnosis of the syndrome and in surgical planning, when indicated. Given the importance of accurate diagnosis of this disease in affected dogs, the current study aimed to carry out an updated literature review addressing the different diagnostic methods for BS. It can then
\end{abstract}


be concluded that the correct identification of brachycephalic syndrome by veterinarians through different diagnostic tools is extremely important to establish an early treatment, minimizing the acute risk of death and increasing the quality of life and welfare of affected animals.

Keywords: Nostril stenosis; Soft palate extension; Laryngoscopy; Rhinoscopy.

\begin{abstract}
Resumen
El reconocimiento temprano del síndrome braquicefálico es de suma importancia para un mejor pronóstico posquirúrgico, y la asociación de varios métodos de investigación es fundamental para su determinación. El diagnóstico del síndrome se basa en una anamnesis precisa junto con la observación de signos clínicos característicos y exámenes complementarios, como exámenes hematológicos y exámenes por imágenes. Entre las pruebas de imagen, se utilizan habitualmente la radiografía, la rinoscopia y la tomografía computarizada, que ayudan tanto en el diagnóstico del síndrome como en la planificación quirúrgica, cuando está indicado. Dada la importancia de un diagnóstico preciso de esta enfermedad en los perros afectados, el presente estudio tuvo como objetivo realizar una revisión de la literatura actualizada que aborde los diferentes métodos de diagnóstico del SB. Entonces se puede concluir que la correcta identificación del síndrome braquicefálico por parte de los veterinarios a través de diferentes herramientas de diagnóstico es de suma importancia para establecer un tratamiento temprano, minimizando el riesgo agudo de muerte y aumentando la calidad de vida y el bienestar de los animales afectados.

Palabras clave: Estenosis de las fosas nasales; Prolongación del paladar blando; Laringoscopia; Rinoscopia.
\end{abstract}

\title{
1. Introdução
}

A síndrome braquicefálica (SB) ou síndrome respiratória obstrutivas dos braquicéfalos (SORB) caracteriza-se por deformidades anatômicas obstrutivas em vias aéreas anteriores primárias que geram alterações secundárias que aumentam o esforço inspiratório aumentando consequentemente a pressão negativa do trato respiratório cranial, região cervical, torácica e abdominal desencadeando lesões no sistema respiratório, cardiovascular e digestório (Packer \& Tivers, 2015; Crane, Rozanski, Abelson \& DeLaforcade, 2017; (Packer, O’Neill, Fletcher \& Farnworth, 2019).

Os componentes obstrutivos primários da SB são a estenose bilateral de narinas, presença de cornetos nasais aberrantes, macroglossia, redução dos espaços naso e orofaríngeo, diminuição do espaço retrofaríngeo, prolongamento de palato mole e hipoplasia de traqueia, que podem levar ao surgimento de alterações secundárias, como espessamento de palato mole, edema e inflamação de orofaringe e nasofaringe, cistos epiglóticos, hiperplasia de tonsilas, sialocele, granulomas de laringe, eversão dos sáculos laríngeos, paralisia ou colapso de laringe, colapso de traqueia e colapso de brônquio principal, podendo levar ao desenvolvimento de um estado grave pelo animal (Emmerson, 2014; Dupré \& Heidenrich, 2016; De Lorenzi, Bertoncello, Mantovani \& Bottero, 2018).

Visando uma melhor resposta clínica, proporcionando assim mais qualidade de vida para os animais acometidos, a instituição do tratamento precoce cirúrgico é imprescindível, sendo necessário o correto diagnóstico para melhor indicação das correções cirúrgicas (Lodato \& Hedlund 2012). A vista disso, este estudo visa realizar uma revisão de literatura abordando as possibilidades diagnósticas desta importante doença na rotina clínica e cirúrgica de pequenos animais.

\section{Metodologia}

Foi realizada revisão de literatura narrativa (Pereira, Shitsuka, Parreira \& Shitsuka, 2018) incluindo artigos científicos publicados e disponíveis nas bases de dados: Capes (Coordenação de Aperfeiçoamento de Pessoal de Nível Superior), Scielo (Scientific Electronic Library Online), Sistema Latino Americano e do Caribe de Informação em Ciências da Saúde (LILACS), PUBMED e Google acadêmico.

Para critérios de inclusão, foram utilizados 25 artigos publicados entre o ano de 2012 e 2021, nas línguas portuguesa e inglesa, com títulos e resumos relacionados à síndrome braquicefálica utilizando os seguintes descritores: síndrome braquicefálica, estenose de narinas e laser cirúrgico. 
Foram excluídos artigos que não apresentavam o resumo, e não abordavam a temática em estudo, bem como artigos de opinião que não estavam apoiados em dados de pesquisa científica ou que não apresentavam suporte de uma coleta sistemática de dados.

Os dados foram coletados através da leitura inicial dos artigos selecionados, sendo em seguida destacadas as informações de maior interesse em cada artigo publicado. Posteriormente foi feita a leitura na íntegra dos artigos referentes ao tema proposto, obtendo-se um conjunto de informações, na qual foram relacionadas com o objetivo, permitindo o desenvolvimento do atual estudo.

\section{Revisão de Literatura}

Para o correto diagnóstico da SB é de extrema relevância o conhecimento mais aprofundado das características intrínsecas das raças (Packer \& Tivers, 2015). De forma geral, a identificação dessa doença obstrutiva se baseia no relato dos tutores, no exame clínico e em exames complementares de imagem e hematologia (Planellas et al., 2012; Dupré \& Heidenreich, 2016; Broux et al., 2018; Kim et al., 2018).

A triagem se inicia a partir de uma rigorosa anamnese, em busca dos sinais clínicos apresentados repetidamente pelo paciente, que muitas vezes não se manifestam no momento da consulta (Emmerson, 2014; Manens et al., 2014). O uso de questionários aplicados aos tutores revelou-se efetivo para a pesquisa da sintomatologia em cães braquicefálicos (Roedler, Pohl \& Oechtering, 2013; Pohl; Roedler \& Oechtering, 2016; Mendes Junior, Silva, Soares \& Almosny, 2017). Porém, apesar de comprovada a importância da identificação dos sinais clínicos pelo tutor, o médico veterinário deve sempre estar atento ao fato de que muitos tutores se adaptam à condição apresentada pelo animal, e passam a não reconhecer a sintomatologia (Liu, Sargan, Adams \& Ladlow, 2015).

O médico veterinário deve proceder a um detalhado exame físico geral, com ênfase na avaliação das narinas, da cavidade oral, do palato mole, da laringe, da traqueia, do pulmão (Packer \& Tivers, 2015; Riggs, Liu, Sutton, Sargan \& Ladlow, 2019). Cabe ressaltar que o exame físico realizado após teste de esforço aumenta a sensibilidade da avaliação do grau de acometimento da SB. Se durante a avaliação ambulatorial o paciente não apresentar sinais clássicos, pode ser realizado um teste de esforço de aproximadamente seis minutos para avaliação da resposta fisiológica do animal ao exercício (Riggs et al., 2019).

Para determinação do grau de estenose de narinas, a visualização direta é o exame de eleição (Davis, Cummings \& Payton, 2017). A inspeção das narinas é simples e deve ser feita levando-se em consideração a oclusão em cada raça (Figura 1). Considera-se aberta a narina quando a parede lateral não toca a medial e levemente estenosada, quando a narina é mais fechada, porém as paredes ainda não se tocam. Na estenose classificada como moderada observa-se que a porção mediodorsal das asas da narina se tocam, porém a região ventral é aberta. No grau mais grave de estenose, tem-se o fechamento total das asas da narina e, geralmente, a respiração do animal é oral. Ressalte-se que esse método de avaliação é preconizado no diagnóstico das obstruções externas à cavidade, mas não é válido para a pesquisa de obstruções intranasais (Liu et al., 2017).

A Figura 1 apresenta a graduação de estenose de narinas nas principais raças braquicefálicas. As narinas abertas são aquelas cuja parede lateral não toca a medial. As levemente estenosadas são aquelas em que a parede lateral ainda não toca a medial. Nas narinas moderadamente estenosadas a parede lateral toca a parede medial da narina na porção dorsal e na porção caudal aberta. As narinas gravemente estenosadas são aquelas em que a parede lateral toca a parede medial da narina na porção dorsal e na porção caudal (Liu et al., 2017). 
Research, Society and Development, v. 10, n. 15, e218101522684, 2021

(CC BY 4.0) | ISSN 2525-3409 | DOI: http://dx.doi.org/10.33448/rsd-v10i15.22684

Figura 1 - Graduação de estenose de narinas nas principais raças braquicefálicas.

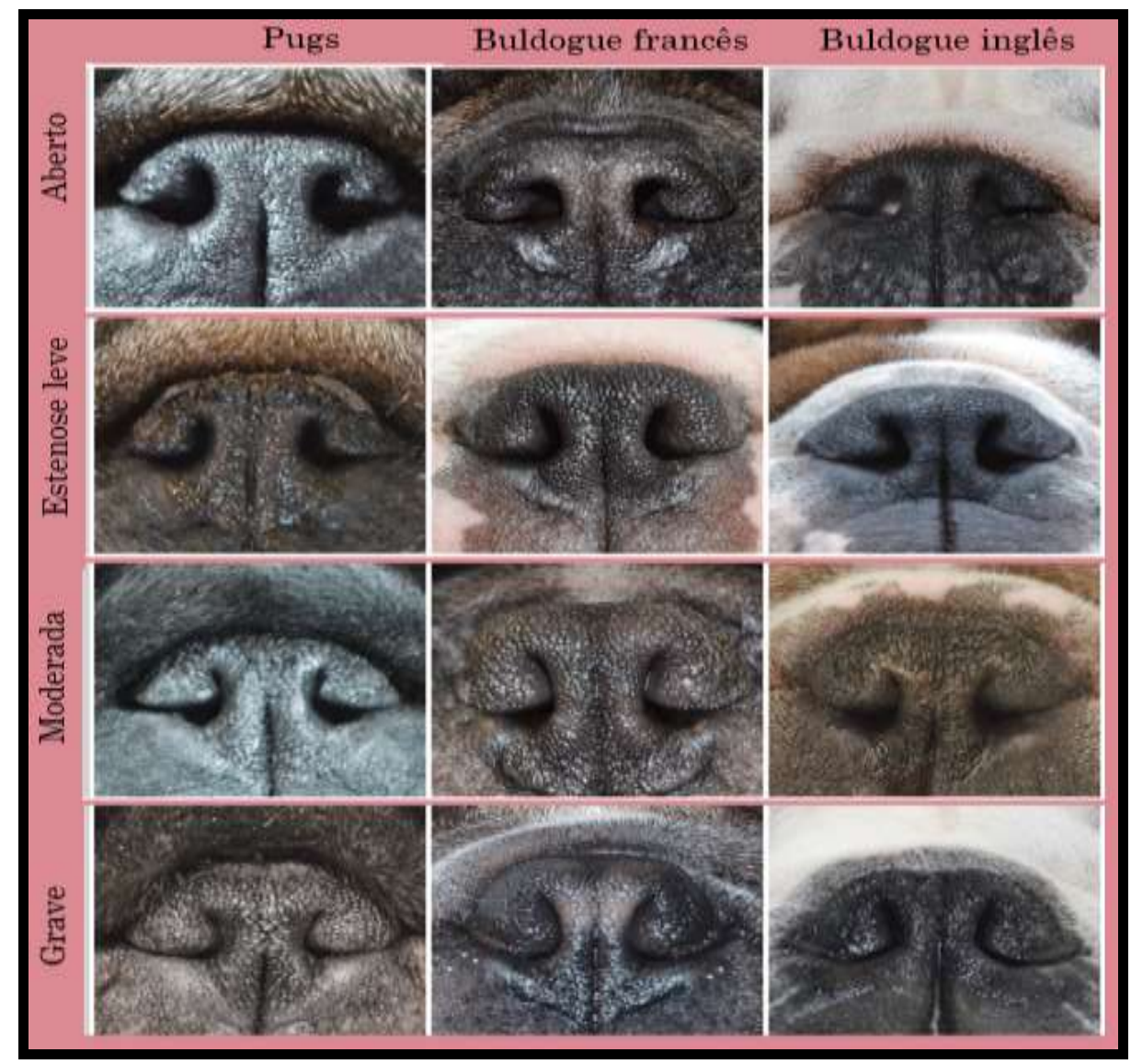

Fonte: Adaptado de Liu et al. (2017).

Clinicamente, o exame do palato mole e da orofaringe é realizado de forma direta, com o paciente anestesiado (Figura 2). Utiliza-se um laringoscópio para compressão da língua e movimentação da epiglote, facilitando assim a visualização do comprimento e da espessura do palato, que não deve ultrapassar a ponta da epiglote e a porção caudal das tonsilas palatinas. Anatomicamente, o prolongamento é qualificado quando se identifica a protusão do palato além da epiglote e das tonsilas (Brockman, Holt \& Haar, 2005). 
Research, Society and Development, v. 10, n. 15, e218101522684, 2021

(CC BY 4.0) | ISSN 2525-3409 | DOI: http://dx.doi.org/10.33448/rsd-v10i15.22684

Figura 2-Realização de laringoscopia em paciente anestesiado para avaliação do palato mole, da região da faringe e da laringe.

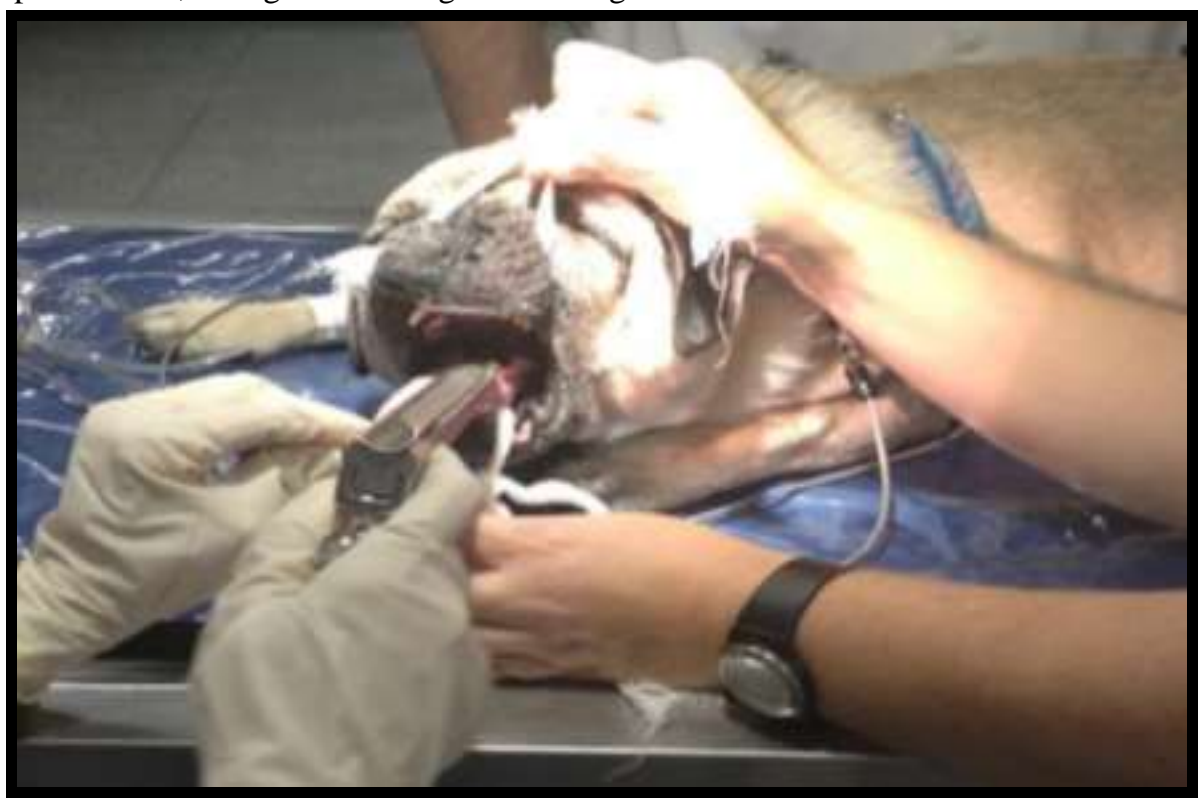

Fonte: Projeto Narizinho, Hospital Universitário de Medicina Veterinária Professor Firmino Mársico Filho - Universidade Federal Fluminense (HUVET- UFF) (2016)

O exame radiográfico é amplamente empregado no diagnóstico da SB. De fácil execução, a radiografia lateral simples de região cervical é um método que pode auxiliar na estimativa do prolongamento e do espessamento do palato e da região da nasofaringe (Figura 3) (Brockman, Holt \& Haar, 2005).

Figura 3 - Radiografia lateral de região cervical de cão portador da síndrome do braquicéfalo. Nota-se o prolongamento e o espessamento do palato mole (seta).

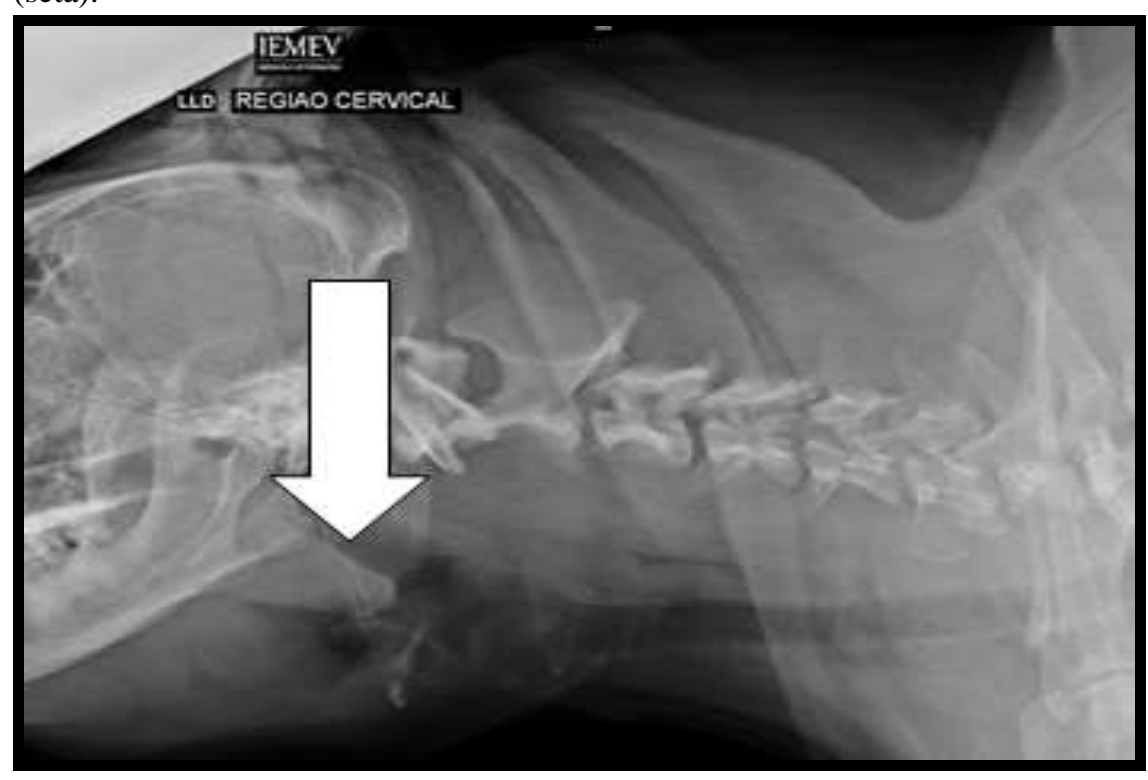

Fonte: IEMEV - Instituto de Especialidades em Medicina Veterinária (2016). 
O exame radiográfico assume ainda importância no diagnóstico da hipoplasia de traqueia (Figura 4), mediante a realização de radiografia lateral da região cervical, traçando-se uma linha imaginária, que se estende da primeira vértebra do manúbrio à primeira vértebra torácica. A medida do diâmetro é feita no ponto em que acontece a interseção desta linha com a traqueia. Quando essa relação é menor que 0,13 no Buldogue inglês e 0,16 no Buldogue francês e no Pug, a traqueia é considerada hipoplásica (Meola, 2013; Heidenreich, Gradner, Kneissl \& Dupre, 2016). As radiografias torácicas também servem como apoio diagnóstico para alterações pulmonares concomitantes, como broncopatia, pneumonia por aspiração e edema pulmonar, comumente encontradas nesses animais (Packer \& Tivers, 2015).

Figura 4 - Radiografia torácica para diagnóstico de hipoplasia de traqueia. T1; Primeira vértebra torácica; TI; Primeira vértebra do manúbrio TD; Interseção da linha imaginária com a traqueia, onde é realizada a medição; Traço preto; Linha imaginária entre TI e T1.

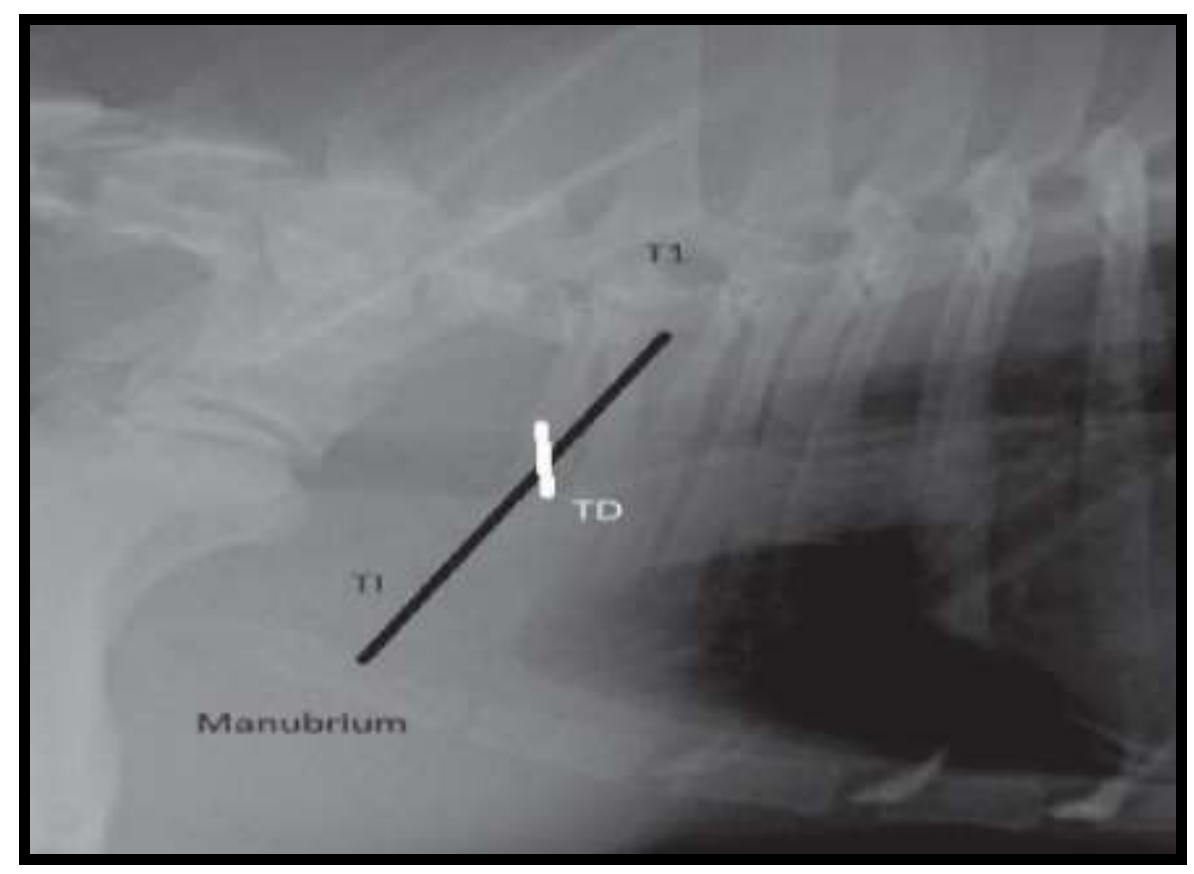

Fonte: Meola (2013).

A rinoscopia (Figura 5) e a tomografia computadorizada são preconizadas para avaliação das variadas obstruções internas da cavidade nasal e da região da faringe. Ressalte-se que a tomografia computadorizada pode ser de extrema valia na obtenção de dados sobre o contato da mucosa nasal e a presença de cornetos aberrantes, podendo ser associada à rinoscopia de vias aéreas anteriores para maior acurácia no diagnóstico das obstruções intranasais (Auger, Alexandre, Beauchamp \& Dunn, 2016). 
Figura 5 - Imagem de rinoscopia retograda da região de nasofaringe posterior de cão da raça Buldogue francês de dois anos de idade. Nota-se estrutura de contornos irregulares e superfície lisa - corneto nasal aberrante posterior (seta). causando obstrucão de coana esauerda.

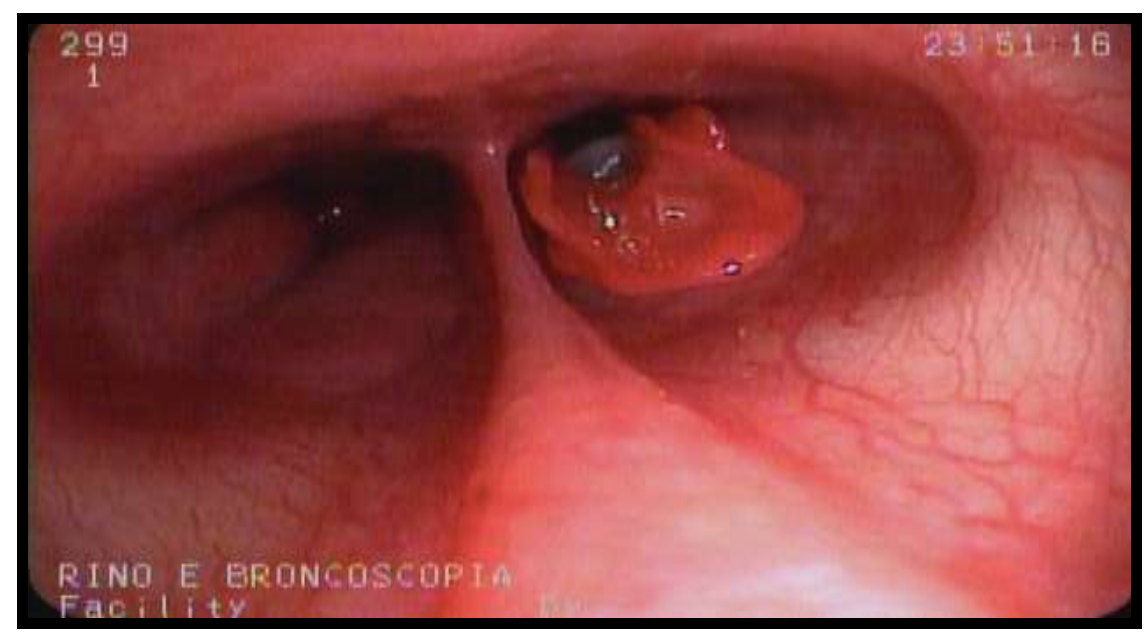

Fonte: Dra . Flávia Toledo (2020).

Oechtering, Pohl, Schlueter e Schuenemann (2016), com o objetivo de averiguar a prevalência das obstruções intranasais nas principais raças braquicefálicas, realizaram os dois exames em cães portadores de dispneia inspiratória grave. O contato íntimo entre as mucosas dos cornetos foi visto no exame endoscópico de $91,7 \%$ deles, que indicou de forma subjetiva uma diminuição no lúmen da nasofaringe. Segundo as imagens tomográficas, todos animais apresentaram crescimentos anormais intracavitários.

A tomografia computadorizada também tem sido utilizada para a apreciação das dimensões do palato mole e da região da oro e nasofaringe (Heidenreich et al., 2016). Atualmente, a ressonância magnética é aplicada, com bons resultados, na avaliação da influência do palato mole na redução do espaço nasofaríngeo (Kim et al., 2018).

Por fim, relata-se o uso da pletismografia barométrica de corpo inteiro (Figura 6) como prova diagnóstica de boa acurácia na pesquisa da SB (Liu et al., 2015; Liu, Adams, Kalmar, Ladlow \& Sargan, 2016; Riggs et al., 2019). A pletismografia barométrica é um exame não invasivo de avaliação da função respiratória, primariamente validado em ratos (Hernandez et al., 2012), e que vem sendo executado experimentalmente em cães e gatos (Liu et al., 2015; Liu et al., 2016; Davis, Cummings \& Payton, 2017; Riggs et al., 2019). O teste é realizado em câmara de acrílico transparente, e os parâmetros respiratórios do paciente são captados por software digital. O teste permite medições quantitativas seguras e repetidas dos ciclos respiratórios em cães não sedados, o que gera resultados mais fidedignos (Liu et al., 2015). 
Figura 6 - Câmara usada para pletismografia barométrica de corpo inteiro com um buldogue francês sendo submetido ao teste.

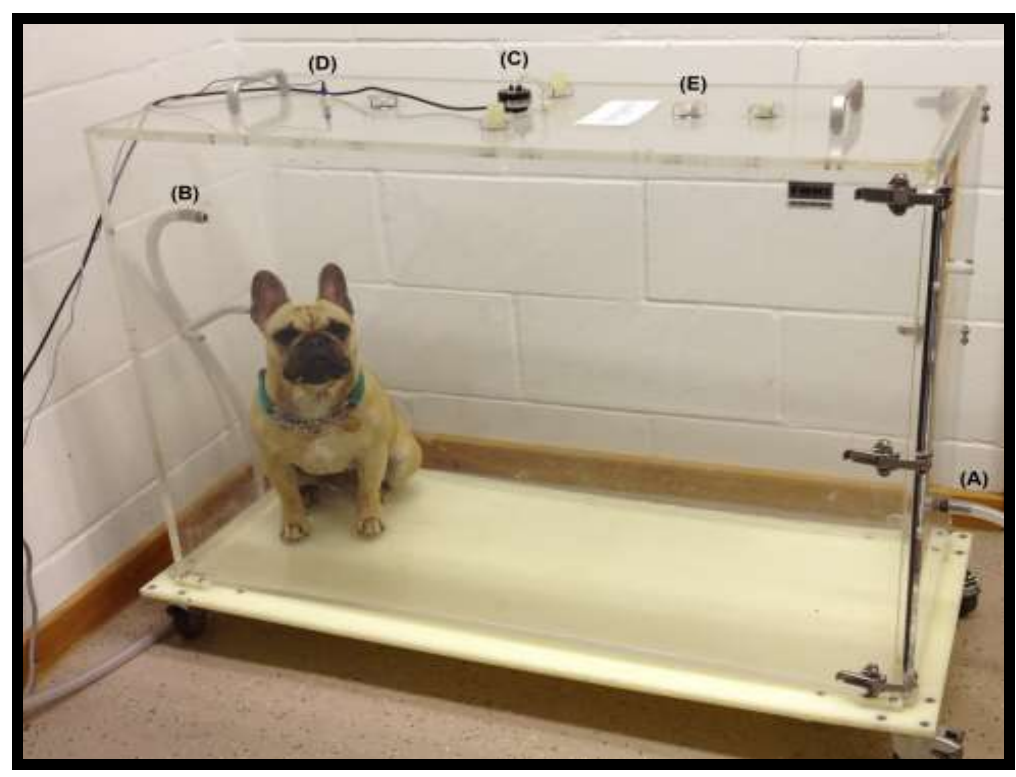

Fonte: Liu et al. (2015).

A pletismografia barométrica realizada em cães braquicefálicos revelou mais alterações respiratórias que os demais cães e demonstrou o aumento da gravidade dos sinais de acordo com o grau obstrutivo de cada animal (Liu et al., 2015). Cães da raça Pug apresentaram mais alterações negativas nos parâmetros aferidos pela pletismografia, indicando que esses animais desenvolvem sinais mais graves de obstrução anatômica. A obesidade e a presença de estenose de narinas também foram correlacionadas de forma direta com a evolução da doença (Liu et al., 2016).

\section{Considerações Finais}

Após levantamento e interpretação dos dados da literatura atual ressalta-se do conhecimento dos diferentes métodos diagnósticos complementares que permitem a identificação precoce da doença pelo médico veterinário para instituição correta do tratamento o mais cedo possível conferindo melhor qualidade de vida e bem estar além de minimizar o risco agudo de óbito nos animais acometidos.

Assim sendo sugere-se a produção de trabalhos que avaliem o conhecimento de médicos veterinários e tutores acerca da síndrome braquicefálica.

\section{Referências}

Auger, M., Alexander, K., Beauchamp, G., \& Dunn, M. (2016). Use of CT to evaluate and compare intranasal features in brachycephalic and normocephalic dogs. The Journal of small animal practice, 57(10), 529-536. https://doi.org/10.1111/jsap.12541

Brockman, D. J., Holt, D. E., \& Haar, G. T. (2018). BSAVA manual of canine and feline head, neck and thoracic surgery (No. Ed. 2). British Small Animal Veterinary Association. https://doi.org/10.22233/9781910443347.6

Broux, O., Clercx, C., Etienne, A. L., Busoni, V., Claeys, S., Hamaide, A., \& Billen, F. (2018). Effects of manipulations to detect sliding hiatal hernia in dogs with brachycephalic airway obstructive syndrome. Veterinary Surgery, 47(2), 243-251. https://doi.org/10.1111/vsu.12735

Crane, C., Rozanski, E. A., Abelson, A. L., \& deLaforcade, A. (2017). Severe brachycephalic obstructive airway syndrome is associated with hypercoagulability in dogs. Journal of Veterinary Diagnostic Investigation, 29(4), 570-573. https://doi.org/10.1177/1040638717703434

Davis, M. S., Cummings, S. L., \& Payton, M. E. (2017). Effect of brachycephaly and body condition score on respiratory thermoregulation of healthy dogs. Journal of the American Veterinary Medical Association, 251(10), 1160-1165.https://doi.org/10.2460/javma.251.10.1160 
Lorenzi, D., Bertoncello, D., Mantovani, C., \& Bottero, E. (2018). Nasopharyngeal sialoceles in 11 brachycephalic dogs. Veterinary Surgery, 47(3), 431-438. https://doi.org/10.1111/vsu.12771

Dupré, G., \& Heidenreich, D. (2016). Brachycephalic Syndrome. The Veterinary clinics of North America. Small animal practice, 46(4), 691-707. https://doi.org/10.1016/j.cvsm.2016.02.002

Emmerson T. (2014). Brachycephalic obstructive airway syndrome: a growing problem. The Journal of small animal practice, 55(11), 543-544. https://doi.org/10.1111/jsap.12286

Heidenreich, D., Gradner, G., Kneissl, S., \& Dupré, G. (2016). Nasopharyngeal dimensions from computed tomography of pugs and French bulldogs with brachycephalic airway syndrome. Veterinary Surgery, 45(1), 83-90.https://doi.org/10.1111/vsu.12418

Hernandez, A. B., Kirkness, J. P., Smith, P. L., Schneider, H., Polotsky, M., Richardson, R. A., ... \& Schwartz, A. R. (2012). Novel whole body plethysmography system for the continuous characterization of sleep and breathing in a mouse. Journal of applied physiology, 112(4), 671-680. https://doi.org/10.1152/japplphysiol.00818.2011

Kim, Y. J., Lee, N., Yu, J., Lee, H., An, G., Bang, S., Chang, J. \& Chang, D. (2018). Three-dimensional volumetric magnetic resonance imaging (MRI) analysis of the soft palate and nasopharynx in brachycephalic and non-brachycephalic dog breeds. Journal of Veterinary Medical Science, 17-0711. https://doi.org/10.1292/jvms.17-0711

Liu, N. C., Adams, V. J., Kalmar, L., Ladlow, J. F., \& Sargan, D. R. (2016). Whole-body barometric plethysmography characterizes upper airway obstruction in 3 brachycephalic breeds of dogs. Journal of veterinary internal medicine, 30(3), 853-865. https://doi.org/10.1111/jvim.13933

Liu, N. C., Sargan, D. R., Adams, V. J., \& Ladlow, J. F. (2015). Characterisation of Brachycephalic Obstructive Airway Syndrome in French Bulldogs Using Whole-Body Barometric Plethysmography. PloS one, 10(6), e0130741. https://doi.org/10.1371/journal.pone.0130741

Liu, N. C., Troconis, E. L., Kalmar, L., Price, D. J., Wright, H. E., Adams, V. J., Sargan, D. R., \& Ladlow, J. F. (2017). Conformational risk factors of brachycephalic obstructive airway syndrome (BOAS) in pugs, French bulldogs, and bulldogs. PloS one, 12(8), e0181928. https://doi.org/10.1371/journal.pone.0181928

Lodato, D. L., \& Hedlund, C. S. (2012). Brachycephalic airway syndrome: management. Compend Contin Educ Vet, $34(8)$, E4.

Manens, J., Ricci, R., Damoiseaux, C., Gault, S., Contiero, B., Diez, M., \& Clercx, C. (2014). Effect of body weight loss on cardiopulmonary function assessed by 6-minute walk test and arterial blood gas analysis in obese dogs. Journal of Veterinary Internal Medicine, 28(2), 371-378. https://doi.org/10.1111/jvim.12260

Mendes Junior, A. F., Silva, G. S., Soares, A. M., \& Almosny, N. R. (2017). Percepção de tutores quanto aos sinais clínicos em cães braquicefálicos portadores de estenose de narina. Enciclopédia Biosfera, 14(26). DOI: 10.18677/EnciBio_2017B46

Meola, S. D. (2013). Brachycephalic airway syndrome. Topics in companion animal medicine, 28(3), 91-96.

Oechtering, G. U., Pohl, S., Schlueter, C., \& Schuenemann, R. (2016). A Novel Approach to Brachycephalic Syndrome. 2. Laser-Assisted Turbinectomy (LATE). Veterinary surgery : VS, 45(2), 173-181. https://doi.org/10.1111/vsu.12447

Packer, R. M., \& Tivers, M. S. (2015). Strategies for the management and prevention of conformation-related respiratory disorders in brachycephalic dogs. Veterinary medicine (Auckland, N.Z.), 6, 219-232. https://doi.org/10.2147/VMRR.S604

Packer, R. M., O’Neill, D. G., Fletcher, F., \& Farnworth, M. J. (2019). Great expectations, inconvenient truths, and the paradoxes of the dog-owner relationship for owners of brachycephalic dogs. PLoS One, 14(7), e0219918. https://doi.org/10.1371/journal.pone.0219918

Planellas, M., Cuenca, R., Tabar, M. D., Bertolani, C., Poncet, C., Closa, J. M., Lorente, J., Cerón, J. J., \& Pastor, J. (2012). Evaluation of C-reactive protein, haptoglobin and cardiac troponin 1 levels in brachycephalic dogs with upper airway obstructive syndrome. BMC veterinary research, 8, 152. https://doi.org/10.1186/1746-6148-8-152

Pohl, S., Roedler, F. S., \& Oechtering, G. U. (2016). How does multilevel upper airway surgery influence the lives of dogs with severe brachycephaly? Results of a structured pre-and postoperative owner questionnaire. The Veterinary Journal, 210, 39-45.https://doi.org/10.1016/j.tvj1.2016.01.017

Riggs, J., Liu, N. C., Sutton, D. R., Sargan, D., \& Ladlow, J. F. (2019). Validation of exercise testing and laryngeal auscultation for grading brachycephalic obstructive airway syndrome in pugs, French bulldogs, and English bulldogs by using whole-body barometric plethysmography. Veterinary Surgery, 48(4), 488-496. https://doi.org/10.1111/vsu.13159

Roedler, F. S., Pohl, S., \& Oechtering, G. U. (2013). How does severe brachycephaly affect dog's lives? Results of a structured preoperative owner questionnaire. Veterinary journal (London, England : 1997), 198(3), 606-610. https://doi.org/10.1016/j.tvj1.2013.09.00 